\title{
Complex profile of multiple hepatobiliary and gastrointestinal complications after hematopoietic stem cell transplantation in a child with Nijmegen breakage syndrome
}

\author{
PRZEMYSŁAW GAŁAZZKA ${ }^{1 *}$, KRZYSZTOF CZYŻEWSKI ${ }^{*}$, ANNA SZAFLARSKA-POPŁAWSKA ${ }^{3}$, \\ ROBERT DĘBSKI ${ }^{2}$, ANNA KRENSKA ${ }^{2}$, JAN STYCZYNSSKI

\begin{abstract}
${ }^{1}$ Department of General and Oncological Surgery for Children and Adolescents, Ludwik Rydygier Collegium Medicum in Bydgoszcz, Nicolaus Copernicus University in Torun, Antoni Jurasz University Hospital No. 1, Bydgoszcz, Poland

${ }^{2}$ Department of Paediatrics, Haematology and Oncology, Ludwik Rydygier Collegium Medicum in Bydgoszcz, Nicolaus Copernicus University in Torun, Antoni Jurasz University Hospital No. 1, Bydgoszcz, Poland

${ }^{3}$ Department of Paediatric Endoscopy and Gastrointestinal Function Testing, Ludwik Rydygier Collegium Medicum in Bydgoszcz,
\end{abstract} \\ Nicolaus Copernicus University in Torun, Antoni Jurasz University Hospital No. 1, Bydgoszcz, Poland
}

"These authors contributed equally to this study.

\begin{abstract}
Patients with Nijmegen breakage syndrome (NBS) can develop life-threatening immunodeficiency, which should be treated with hematopoietic stem cell transplantation (HSCT). We report the case of a 14-year-old girl with NBS who due to an increasing number of severe complications was referred for HSCT from a matched unrelated donor. After reduced-intensity conditioning and transplantation of peripheral blood hematopoietic cells, during the early post-transplant period (days 0-30), the girl suffered from severe mucositis, fever episodes, mild acute renal injury and facial vasculitis. All these complications were managed successfully. During the intermediate post-transplant period (days 30-100) a number of hepatic and gastrointestinal complications occurred, including cholecystitis, cholelithiasis with choledocholithiasis, pancreatitis as well as acute bleeding from the lower gastrointestinal tract caused by rectal and recto-sigmoid junction ulcers. All the obstacles were obviously attributable both to the primary congenital disease, its complications, and transplantation itself. We overcame these complications and treated the patient with the best possible and safe methods. The multidisciplinary approach based on combined surgical, endoscopic and conservative management of multiple post-transplant complications was successful for the patient.
\end{abstract}

Key words: hematopoietic cell transplantation, cholecystitis, cholelithiasis, choledocholithiasis, pancreatitis, gut ulcer.

(Centr Eur J Immunol 2019; 44 (3): 327-331)

\section{Introduction}

Nijmegen breakage syndrome (NBS) is an autosomal recessive chromosomal instability disorder. Majority of patients affected are of Slavic origin and share the same founder mutation of 657 del 5 within the NBN gene encoding protein involved in DNA double-strand breaks repair. Clinically, this is characterized by a microcephaly, immunodeficiency and a high incidence of pediatric malignancies, mostly lymphomas and leukemias [1]. Recurrent episodes of pneumonia may result in respiratory failure and early death [2]. The major therapeutic issue in
NBS patients is a prophylaxis of infections based on intravenous immunoglobulin supplementation together with administration of antifungal and antibacterial agents. In severe cases of immunodeficiency, hematopoietic stem cell transplantation (HSCT) with reduced-intensity conditioning (RIC) should be considered, however current transplant experience in patients with NBS is very small [3].

\section{Case report}

A 14-year-old girl with NBS presented with a typical phenotype and genotype (homozygotic NBN gene mu-

Correspondence: Prof. Jan Styczyński, Department of Paediatrics, Haematology and Oncology, Ludwik Rydygier Collegium Medicum in Bydgoszcz, Nicolaus Copernicus University in Torun, Antoni Jurasz University Hospital No. 1, 9 Marii Skłodowskiej-Curie St., 85-094 Bydgoszcz, Poland, e-mail: jstyczynski@cm.umk.pl Submitted: 18.04.2018; Accepted: 24.05.2018. 
tation 657del5) has suffered since early childhood from acute and chronic infections of the respiratory tract, paranasal sinuses, cryptococcal meningoencephalitis, chronic bronchitis, chronic pneumonia, symptomatic Epstein-Barr virus (EBV) reactivation, hepatitis of unknown etiology with cholestasis, and skin infections including disseminated actinomycosis and non-tuberculosis granulomas. She has also been diagnosed and treated for secondary hemophagocytic lymphohistiocytosis (HLH), complicated with facial vasculitis, hypertension and cardiomyopathy. Additionally, she had a tri-lineage hematological insufficiency and hypogammaglobulinemia $(\operatorname{IgA}<10 \mathrm{mg} / \mathrm{dl}$, $\mathrm{IgG} 85 \mathrm{mg} / \mathrm{dl}$ ). Absolute numbers of lymphocytes showed combined B- and T-cell immunodeficiency: $0.266 \mathrm{G} / \mathrm{l}$ for CD3; $0.084 \mathrm{G} / 1$ for CD4; $0.140 \mathrm{G} / 1$ for CD8 and $0.077 \mathrm{G} / \mathrm{l}$ for CD19.

Due to an increasing number of life-threatening infectious complications, confirmed progressing immunodeficiency and marrow hypocellularity, she was referred for HSCT from a matched unrelated donor. On admission, she was in overall poor clinical condition, with Lansky performance score of 80 , infection of the skin, symptoms of chronic pulmonary disease with various pulmonary rhonchi, oxygen saturation of $94 \%$, and abnormal liver function tests.

The preparative RIC regimen included fludarabine (30 mg/m²/day; days from -9 to -5 ), cyclophosphamide ( $5 \mathrm{mg} / \mathrm{kg}$ bw/day; days from -5 to -2 ) and antithymocyte globulin (total $8 \mathrm{mg} / \mathrm{kg}$ bw divided between three consecutive days from -3 to -1 ). Due to previous infections, she was on continuous therapy with antibiotics. Other anti-infective prophylaxis included posaconazole and acyclovir. She received also prophylaxis against veno-occlusive dis- ease with defibrotide. The graft included peripheral blood stem cells from HLA-matched, AB0/Rh-matched woman $\left(11.6 \times 10^{8} \mathrm{NMC} / \mathrm{kg}\right.$ bw; $7.8 \times 10^{6} \mathrm{CD} 34+$ cells $/ \mathrm{kg}$ bw $)$. Prophylaxis against graft-versus-host disease (GVHD) included cyclosporine A and mycophenolate mofetil, however due to deterioration of parameters of the renal function, cyclosporine was converted to sirolimus, which in turn was converted to methylprednisolone, due to facial vasculitis (Fig. 1). Additionally, at day +5 rituximab was administered prophylactically against EBV reactivation and development of post-transplant lymphoproliferative disorder (EBV-PTLD).

During an early post-transplant period she was in otherwise good condition, however she suffered from severe mucositis, fever episodes, and necessity of total parenteral nutrition (TPN). Hematological and platelet recovery occurred at days +12 and +25 , respectively. She was discharged from the transplant ward at day +28 with $49 \%$ donor chimerism, normal hematopoiesis in bone marrow, and no CMV or EBV viremia.

At day +35 she complained of acute abdominal pain accompanied by significant elevation of gamma glutamyl transpeptidase (3673 U/1), alkaline phosphatase activity (643 U/l) and a total and conjugated bilirubin level (5.17 mg/dl and $4.15 \mathrm{mg} / \mathrm{dl}$, respectively). On magnetic resonance (MRI) cholangiopancreatography, the common bile duct (CBD) was widened with a diameter of $6 \mathrm{~mm}$ and in the distal part of CBD there were bile deposits of $5 \times 3 \mathrm{~mm}$ size. She was qualified to urgent endoscopic retrograde cholangiopancreatography (ERCP). After sphincterotomy, removal of bile deposits was carried on with endoscopic basket. Additionally a $5 \mathrm{~cm} 7 \mathrm{~F}$ prosthesis was inserted into the distal CBD (Fig. 2).
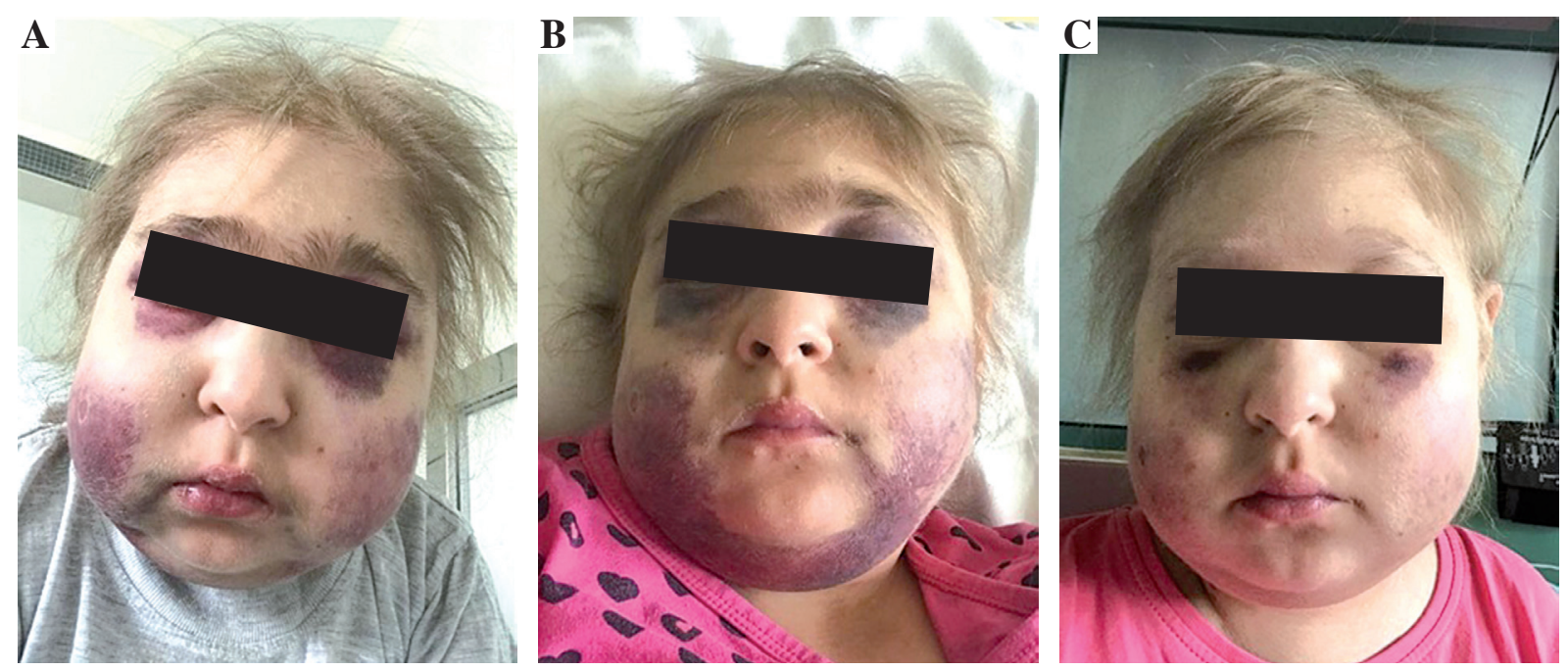

Fig. 1. Facial vasculitis and symptoms of Cushing syndrome in the NBS patient at days: $+19,+20$ and +26 after transplantation 

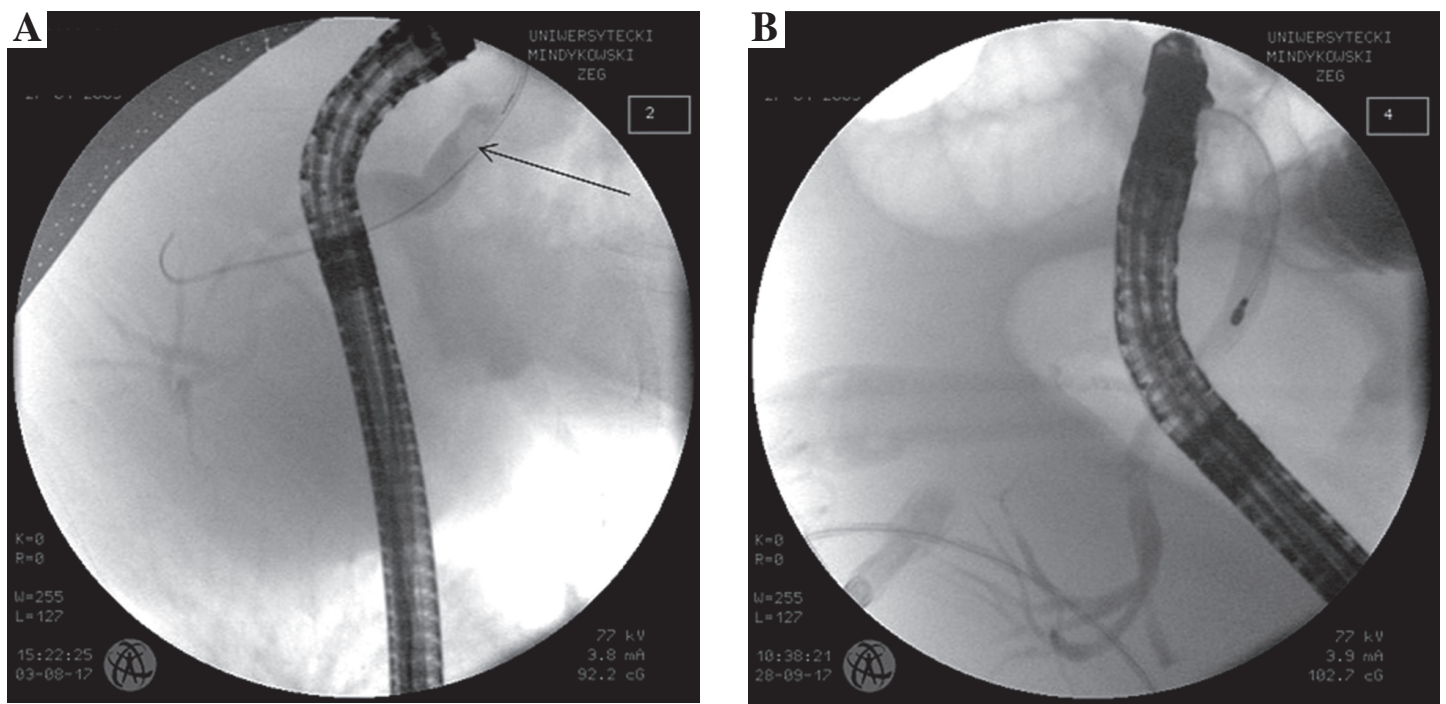

Fig. 2. ERCP showing a common bile duct $($ day +35$)$ : A) mildly dilated duct with biliary stone (black arrow) before extraction, B) with no signs of dilation after stone extraction (6 weeks later)

She was initially planned for early (within 7 days) CBD prosthesis removal, however this approach was postponed because of post-ERCP pancreatitis development with elevation of serum and urine amylase activity (2701 U/1 and $3803 \mathrm{U} / 1$, respectively). In the subsequent MRI examination, signs of pancreatic edema and irregularity of pancreatic borders were noted. Despite the presence of peripancreatic fluid collections, stranding fat densities and temporary signs of paralytic ileus she was successfully treated with TPN, fluid resuscitation and broad-spectrum antibiotics. She was qualified to elective laparoscopic cholecystectomy, which was performed 6 weeks after ERCP. On laparoscopy a thickened, callous wall of the gall bladder was revealed. Liver surface appeared to be nodular with increased consistency. Due to the problems with clear delineation of gall bladder bed, the lack of possibility of proper identification of hepatic hilum structures and bleeding, the procedure was converted to the open cholecystectomy. After gallbladder and biliary stent removal, a careful hemostasis of gallbladder fossa was performed using argon beam coagulation and 4DryField starch powder. In the early postoperative course an increased amount of drained fluid was observed but drainage was removed on the third postoperative day and spontaneous biliary flow without any complications was obtained. Due to subsequent transient elevation of serum amylase and poor wound healing she was successfully managed conservatively with vancomycin and ceftriaxone. Further postoperative course was uneventful.

Two weeks later she was admitted to hospital with mild acute lower gastrointestinal bleeding. Stool samples were tested negative for rotavirus, adenovirus and norovirus, but positive for Campylobacter jejuni and Clostridium

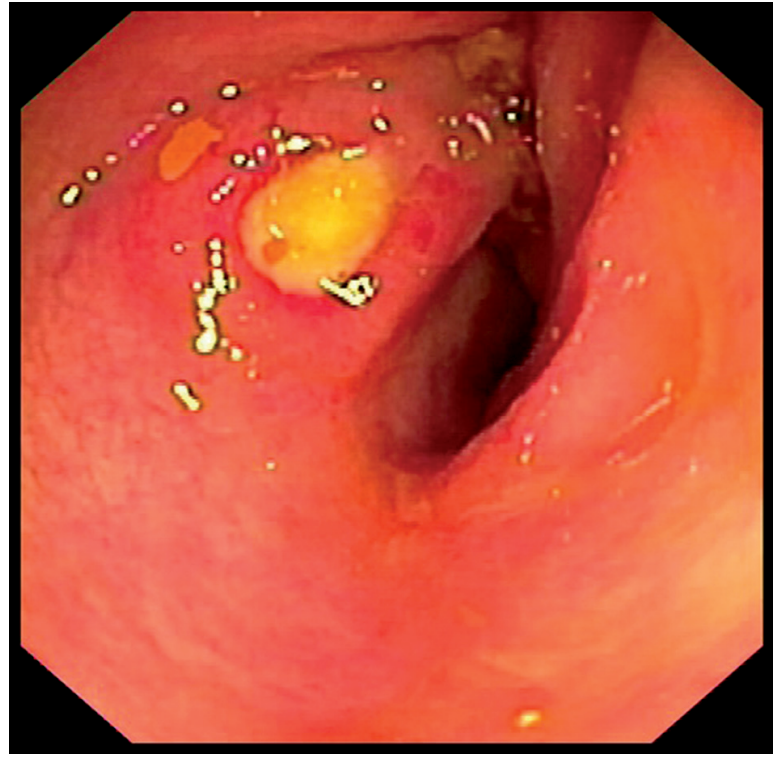

Fig. 3. Endoscopic picture showing deep $2 \mathrm{~cm}$ in diameter crater-like ulcer located at the recto-sigmoid junction covered by yellowish slough with inflamed surrounding mucosa $($ day +104$)$

difficile. Blood CMV and EBV reactivation were also ruled out. The ileocolonoscopy revealed a 3-4 cm crater-like ulcer located 2-3 cm from the anal margin, which was covered by yellowish slough surrounded by reactive mucosa. Another ulcer was found at the recto-sigmoid junction (Fig. 3). Microscopically, lymphocytic and neutrophilic mucosa infiltrate that focally infiltrated crypt epithelium with mild crypt distortion and signs of regeneration 
Table 1. Complications before and after hematopoietic stem cell transplantation

\begin{tabular}{ll}
\hline Age/time & \multicolumn{1}{c}{ Complications } \\
\hline $3-13$ years & $\begin{array}{l}\text { Chronic bronchitis, chronic pneumonia, chronic sinusitis, disseminated skin actinomycosis, skin granulomas, liver } \\
\text { insufficiency with cholestasis }\end{array}$ \\
\hline 13 years & $\begin{array}{l}\text { CNS cryptococcosis (treatment in Intensive Care Unit), secondary hemophagocytic lymphohistiocytosis, vasculitis, bone } \\
\text { marrow hypocellularity }\end{array}$ \\
\hline 14 years & Combined B/T-cell immunodeficiency, bone marrow failure, skin dermatofibroma \\
\hline HSCT (day $\mathbf{0})$ \\
\hline Day $+10 \quad$ Severe mucositis \\
\hline Day +19 & Deterioration of renal function, facial vasculitis \\
\hline Day +35 & Symptomatic choledocholithiasis \\
\hline Day +42 & Pancreatitis \\
\hline Day +77 & Recurrent cholelithiasis with cholecystitis \\
\hline Day +91 & Acute skin graft-versus-host disease II $^{\circ}$ \\
\hline Day +99 & Acute bleeding from the lower gastrointestinal tract: rectal and recto-sigmoid junction ulcers \\
\hline Day +200 & No complaints; overall good condition \\
\hline HSCT - hematopoietic stem cell transplantation, CNS - central nervous system
\end{tabular}

were present in the entire colon. Based on histopathology, CMV infection and GVHD in the colon were excluded. The girl was treated with intravenous azithromycin and orally budesonide and mesalamine. A clinical and endoscopic improvement was noted at the second endoscopy performed four weeks later.

Three month later no complaints were reported (Table 1). There were symptoms of immunological recovery, and the girl presented an increasing donor chimerism $(63 \%)$.

\section{Discussion}

The first report on treatment with HSCT in children with NBS was reported in 2010 [4], and up to 2017 data on a total number of 19 patients were published, including only three without underlying malignancy [3-6]. Recently, two analyses with data on 26 and 35 patients, respectively, were reported [7, 8]. In NBS patients without malignancy, the crucial pre-transplant problem includes development of severe immunodeficiency followed by infections. This was the case of our patient, who over the years developed symptomatic severe combined immunodeficiency syndrome.

The peritransplant period was typical, with manageable complications including myelosuppression, severe mucositis, facial vasculitis, mild acute kidney injury and neutropenic fever. Nevertheless, she was discharged from the transplant unit at day +28 in overall good condition. However, after day +30 , a number of severe complications have developed including choledocholithiasis and recto-sigmoid ulcers.

Data on cholelithiasis after HSCT in the early post-transplant period are very scanty [9], and there is no report on cholelithiasis in patients with NBS after HSCT. The well-known risk factors associated with gallstones in pediatric population include autologous transplant, unrelated donor, grade 3 to 4 acute GVHD, chronic GVHD, second transplant, diabetes, and estrogen therapy [10]. Hemolysis is another possible risk factor [11]. None of these risk factors was present in our patient at day +35 . Importantly, our patient was diagnosed for cholestasis after hepatitis of unknown etiology, already before HSCT. Non-malignant disease, mucositis, neutropenic fever and the necessity of TPN were additional risk factors. Clinical data show that children who underwent HSCT for malignancy showed a significantly lower risk of cholelithiasis compared with those treated for bone marrow failure (7.4\% vs $27 \%)$ [12].

In the late post-transplant period, most children (85\%) with gallstones after HSCT usually did not require surgical intervention, and strategy of non-operative management for asymptomatic cholelithiasis in this highly selected group of patients is supported [12]. According to other authors, surgical exploration and cholecystectomy still remain an important approach even in the case of acalculous acute cholecystitis [13].

The main difficulties in the management of our patient was the necessity of invasive diagnosis and treatment in the early post-transplant period, gastric endoscopy, ileocolonoscopy, endoscopic retrograde cholangiopancreatography, and open cholecystectomy. We had to answer the questions on selection of either conservative or surgical treatment of cholelithiasis, and either one or two interventions. The approach of two surgical interventions was successful for the patient. We decided to perform "delayed cholecystectomy" about 6 weeks after an episode of acute cholecystitis, obstructive jaundice and post-ECPW pancre- 
atitis. This was a sufficient approach for the improvement in the general status of the patient and local conditions. We believe that an invasive strategy, consisting of close monitoring and early laparotomy combined with vigorous supportive therapy, should be used when dealing with suspected gastrointestinal complications in patients with hematological malignancies. Our patient presented acute symptoms and complications of cholelithiasis. In our opinion, the clinical picture of cholecystitis in our patient was progressive. This is why despite poor general condition of the patient, cholecystectomy should be performed in case of urgent indications regardless of anemia, granulocytopenia and thrombocytopenia, which should be corrected as much as possible.

Endoscopic sphincterotomy after biliary pancreatitis may suffice for early treatment in lieu of cholecystectomy to prevent recurrent symptoms before delayed cholecystectomy: patients who underwent ERCP with sphincterotomy decreased their risk of readmission from $24 \%$ to $10 \%$ and their risk of developing recurrent biliary pancreatitis from $9 \%$ to $1 \%[14,15]$.

Related acute abdominal complications often need emergency surgical treatment with a high rate of mortality. In these patients the surgical strategy is complex and hard to schematize. When deciding on the surgical strategy in treating of acute abdominal complications in the patient at early phase after HSCT, the surgeon must consider that surgical intervention is indicated only after unsuccessful medical treatment. Proper cooperation of a multi-disciplinary team plays a crucial role in the preparation and stabilization of patients' general status. Obviously, post-transplant neutropenia, thrombocytopenia, immune suppression or positive blood cultures are adverse prognostic factors.

\section{Conclusions}

In conclusion, the key issue for our patient in the intermediate post-transplant period between days +30 and +100 were hepatic and pancreatic complications. All the obstacles were obviously attributable both to the primary congenital disease, its complications, and transplantation itself. We overcame these complications and treated the patient with the best possible and safe approach. Finally, we solved also the nature of recto-sigmoid ulcers, which ex iuvantibus has proven to be an unspecific inflammation of the gastrointestinal tract. In the meantime we excluded gastrointestinal GVHD, CMV infection, and late toxicity. This multidisciplinary approach based on combined surgical, endoscopic and conservative management of multiple post-transplant complications was successful for the patient.

The authors declare no conflict of interest.

\section{References}

1. Pastorczak A, Szczepanski T, Mlynarski W (2016): Clinical course and therapeutic implications for lymphoid malignancies in Nijmegen breakage syndrome. Eur J Med Genet 59: 126-132.

2. Varon R, Demuth I, Chrzanowska KH (2017): Nijmegen Breakage Syndrome. In: Gene Reviews, Adam MP, Ardinger HH, Pagon RA, et al. (eds.). University of Washington, Seattle 1993 (updated 2017).

3. Wolska-Kusnierz B, Gregorek H, Chrzanowska K, et al. (2015): Nijmegen Breakage Syndrome: clinical and immunological features, long-term outcome and treatment options - a retrospective analysis. J Clin Immunol 35: 538-549.

4. Albert MH, Gennery AR, Greil J, et al. (2010): Successful SCT for Nijmegen Breakage Syndrome. Bone Marrow Transplant 45: 622-626.

5. Stajner T, Vasiljevic Z, Vujic D, et al. (2013): Atypical strain of Toxoplasma gondii causing fatal reactivation after hematopoietic stem cell transplantion in a patient with an underlying immunological deficiency. J Clin Microbiol 51: 2686-2690.

6. Wozniak M, Krzywon M, Holda MK, et al. (2015): Reduced-intensity conditioning umbilical cord blood transplantation in Nijmegen Breakage Syndrome. Pediatr Transplant 19: E51-55.

7. Slack J, Albert MH, Balashov D, et al. (2018): Outcome of hematopoietic cell transplantation for DNA double-strand break repair disorders. J Allergy Clin Immunol 141: 322-328.e10.

8. Deripapa E, Balashov D, Rodina Y, et al. (2017): Prospective study of a cohort of Russian Nijmegen Breakage Syndrome patients demonstrating predictive value of low kappa-deleting recombination excision circle (KREC) numbers and beneficial effect of hematopoietic stem cell transplantation (HSCT). Front Immunol 8: 807.

9. Alnusair MM, DeMagalhaes-Silverman M, Silverman WB (2006): The role of ERCP in patients with pancreatico-biliary problems in the setting of hematopoietic stem cell transplant. Gastrointest Endosc 63: 655-659.

10. Hoffmeister PA, Storer BE, McDonald GB, et al. (2014): Gallstones in pediatric hematopoietic cell transplant survivors with up to 40 years of follow-up. J Pediatr Hematol Oncol 36: 484-490.

11. Andoh A, Hodohara K, Inoue T, et al. (2000): ALL complicated by obstructive jaundice due to choledocholithiasis after unrelated bone marrow transplantation. Rinsho Ketsueki 41: 612-614.

12. Safford SD, Safford KM, Martin P, et al. (2001): Management of cholelithiasis in pediatric patients who undergo bone marrow transplantation. J Pediatr Surg 36: 86-90.

13. Kobbe G, Heyll A, Zodler T, et al. (1997): Acute acalculous cholecystitis complicating oral recontamination after allogeneic bone marrow transplantation. Oncol Rep 4: 823-824.

14. van Baal MC, Besselink MG, Bakker OJ, et al. (2012): Timing of cholecystectomy after mild biliary pancreatitis: a systematic review. Ann Surg 255: 860-866.

15. Eachempati SR, Cocanour CS, Dultz LA, et al. (2014): Acute cholecystitis in the sick patient. Curr Probl Surg 51: 441-466. 